\title{
Analysis of ZIP Load Modeling in Power Transmission System
}

\author{
Seshapalli Sai Ram ${ }^{*}$, Suresh Babu Daram ${ }^{2}$, P. S. Venkataramu ${ }^{3}$ \\ and M. S. Nagaraj ${ }^{4}$ \\ ${ }^{1 *} P G$ Student, ${ }^{2}$ Assistant Professor, Dept. of Electrical \& Electronics Engineering, \\ Sree Vidyanikethan Engineering College, Tirupati \\ ${ }^{3}$ Director IQAC, REVA University, Bangalore \\ ${ }^{4}$ Professor \& Head, Dept. of Electrical \& Electronics Engineering \\ Bapuji Institute of Engineering \& Technology, Davangere \\ ${ }^{1 *}$ seshapallisairam@gmail.com, ${ }^{2}$ sureshbabudaram@gmail.com
}

\begin{abstract}
A Static load model is a relation between measured voltage at a bus, active and reactive power consumed by the load connected at an instant. These models have an exponential or polynomial representation of load. In this paper, the load modeling is considered for constant impedance (Z), constant current (I), constant power (P) and ZIP. The proposed model is incorporated in Newton-Raphson (NR) power flow solution method and analyzed with a MATLAB program. Newton-Raphson $(N-R)$ power flow technique is used for analyzing various load models on the transmission system. The proposed algorithm is tested on a standard 2-machine 5-bus system using MATLAB.
\end{abstract}

Keywords: Load modelling, Constant Z, Constant I, Constant P, ZIP load modelling, Load flow studies

\section{Introduction}

The estimation of a suitable load model is a very complicated task due to the fact that the load characteristics change during a day, days of the week, and seasons. Furthermore, there is a natural load fluctuation, which makes the load modeling even more difficult. Two approaches have been used as tools develop load model. The first one is the component-based approach, which depends on the knowledge of individual components. The second one is the measured-based approach, is based on the analysis of load behavior when subjected to voltage variations, which can be performed by using tap commutations, and does not require the knowledge of the physical characteristics of the load.

Load modeling has been studied since a long time ago to improve the accuracy of power system analysis. Load model is classified into two types those are static load model and Dynamic load model [1].

The following are the advantages of load modeling.

- The variation of power demand with voltage enables better control capacity.

- The calculation of active and reactive power demand at respective buses.

- Control of over and under voltage at load bus.

- Minimization of losses.

- Improvement in voltage profile.

Received (January 6, 2018), Review Result (March 13, 2018), Accepted (April 2, 2018)

* Corresponding Author 
The static and dynamic load models are classified according to the effect of voltage on the load. The load variation depends only on the instantaneous voltage input and is related to the preceding voltage input. The representative static load model is a polynomial-based model which is composed of constant impedance characteristics, constant current characteristics, and constant power characteristics. That static model is also known as ZIP model [2] and is often expand to static load model with frequency characteristics using proportion coefficient. Using ZIP and exponential load models critical points are considered. Composite load models [3], [4] are developed based on on-line measurement data from the practical power system. The ZIP load model has been extensively studied. This is widely applied in composite load models that could maintain constant impedance, constant current and constant power. Composite load model is real and reactive power behavior during the system disturbance [5].

In [6], [7] dynamic load models are more complex because the response of the loads to voltage and frequency variations is a lot faster. The static models used before will be ineffective models in the fast response case. However, the detecting of parameters, in this case, can still rely on measured based and component-based approaches. More accurate measurements [8] need to be taken as the system's response changes rapidly. Due to the complexity, long-term stability, inter-area oscillations, voltage stability become the important criterion to be modeled.

The Power flow study is the main objective of a power system to determine the steady state operating conditions. Steady state operating conditions of a power system can be obtained by calculating active and reactive power flow in the power network [9] and by calculating the magnitudes and angles of the voltage at different nodes of the power network. The main advantage of the Newton Raphson method is its quadratic rate of convergence, which is faster than any other power flow method.

This paper is organized as follows. Firstly, static load models describe in 2. Constant impedance model incorporated to the newton-raphson method described in 3.1. The Constant current model incorporated to newton-raphson method describes in 3.2. Constant power incorporated to newton-raphson method describes in 3.3. ZIP incorporated in newton-raphson method describes in 3.4. A Proposed algorithm explained in 4. Finally, case study simulations result and discussions performed in 5.

\section{Static Load Models}

Different load models would greatly affect power voltage stability analysis. Static load models do not vary with time. In these models, active and reactive power loads are expressed as exponentials and polynomials of voltage and frequency. The different static load models are discussed in following subsections.

\subsection{Exponential Load Model}

The exponential load model for real and reactive power at load bus is represented by a below.

$$
\begin{aligned}
& P=P_{\mathrm{o}}\left(\frac{V}{V_{\mathrm{O}}}\right)^{a} \\
& Q=Q_{0}\left(\frac{V}{V_{0}}\right)^{b}
\end{aligned}
$$

$P_{0}, Q_{0}, V_{0}$ are the initial values of real power, reactive power and voltage at a load bus respectively.

a, b- load parameters of this model, the value of a and b vary between 0 to 2 . 


\subsection{Polynomial Load Model}

This model [10] also called ZIP load model. Z stands for constant impedance, I represent constant current and $P$ refers to constant power. The polynomial model for active and reactive power are given in equation (3) and (4).

$$
\begin{gathered}
P=P_{i}\left\lfloor P_{1} \bar{V}^{2}+P_{2} \bar{V}+P_{3}\right\rfloor \\
Q=Q_{i}\left\lfloor Q_{1} \bar{V}^{2}+Q_{2} \bar{V}+Q_{3}\right\rfloor \\
\text { Here } \bar{V}=\frac{V}{V_{0}}
\end{gathered}
$$

Here, $P_{i}, Q_{i}$ and $V_{0}$ are the nominal values of the load active, reactive power and voltage.

We can obtain the parameters based on the type of load as follows:

2.2.1. Constant Impedance Load Model: $P_{1}=Q_{1}=1 . P_{2}, Q_{2}, P_{3}, Q_{3}=0$. The active and reactive powers are proportional to voltage squared. Then $P_{1}+P_{2}+P_{3}=1$, $Q_{1}+Q_{2}+Q_{3}=1$. Substitute above conditions in equation (3) and (4).

$$
\begin{gathered}
P=P_{i} V^{2} \\
Q=Q_{i} V^{2}
\end{gathered}
$$

2.2.2. Constant Current Load: $P_{2}=Q_{2}=1 . P_{1}, Q_{1}, P_{3}, Q_{3}=0$ The active and reactive powers are proportional to voltage. Then $P_{1}+P_{2}+P_{3}=1, Q_{1}+Q_{2}+Q_{3}=1$. Substitute above conditions in equations (3) and (4).

$$
\begin{gathered}
P=P_{i} V \\
Q=Q_{i} V
\end{gathered}
$$

2.2.3. Constant Power Load: $P_{3}=Q_{3}=1: P_{2}, Q_{2}, P_{1}, Q_{1}=0$. The active and reactive powers are independent of voltage. Then $P_{1}+P_{2}+P_{3}=1, Q_{1}+Q_{2}+Q_{3}=1$. Substitute above conditions in equations (3) and (4).

$$
\begin{array}{r}
P=P_{i} \\
Q=Q_{i}
\end{array}
$$

Most of the loads can be represented as some combination of the ZIP model, with different parameters reflecting the composition. Constant power loads lead to stability problems because there is a tendency to increase the current, in order to maintain constant power even though voltage drops. This can lead to a further drop in the voltage. Constant impedance loads, on the other hand tend to damp voltage oscillations.

\section{Polynomial Load Models are Incorporated with a Newton-Raphson Method}

In this section, we are going to explain how polynomial model incorporated in load flow analysis. 


\subsection{Constant Impedance (Z) Incorporated with Newton-Raphson Method:}

Newton Raphson [11] represents both active and reactive powers and incorporated in equation (5) and (6).

$$
\begin{aligned}
& P_{Z}=\left[\sum_{j=1}^{n}\left|V_{i}\right|\left|V_{j} \| Y_{i j}\right| \cos \left(\theta_{i j}-\delta_{i}+\delta_{j}\right)\right] V_{i}^{2} \\
& Q_{Z}=\left[-\sum_{j=1}^{n}\left|V_{i}\right|\left|V_{j}\right|\left|Y_{i j}\right| \sin \left(\theta_{i j}-\delta_{i}+\delta_{j}\right)\right] V_{i}^{2}
\end{aligned}
$$

The diagonal and the off-diagonal elements of active power are represented below.

$$
\begin{aligned}
& \frac{\partial P_{Z}}{\partial \delta_{i}}=\sum_{j \neq i}\left|V_{i}^{3} \| V_{j}\right|\left|Y_{i j}\right| \sin \left(\theta_{i j}-\delta_{i}+\delta_{j}\right) \\
& \frac{\partial P_{Z}}{\partial \delta_{j}}=-\left|V_{i}^{3}\right|\left|V_{j}\right|\left|Y_{i j}\right| \sin \left(\theta_{i j}-\delta_{i}+\delta_{j}\right) j \neq i \\
& \frac{\partial P_{Z}}{\partial\left|V_{i}\right|}=4\left|V_{i}^{3}\right|\left|Y_{i i}\right| \cos \theta_{i i}+\sum_{j \neq i} 3\left|V_{i}^{2}\right|\left|V_{j}\right|\left|Y_{i j}\right| \cos \left(\theta_{i j}-\delta_{i}+\delta_{j}\right) \\
& \frac{\partial P_{Z}}{\partial\left|V_{j}\right|}=\left|V_{i}^{3}\right|\left|Y_{i j}\right| \cos \left(\theta_{i j}-\delta_{i}+\delta_{j}\right) j \neq i
\end{aligned}
$$

The diagonal and the off-diagonal elements of reactive power are represented below.

$$
\begin{aligned}
& \frac{\partial Q_{Z}}{\partial \delta_{i}}=\sum_{j \neq i}\left|V_{i}^{3}\right|\left|V_{j}\right||Y i j| \cos \left(\theta_{i j}-\delta_{i}+\delta_{j}\right) \\
& \frac{\partial Q_{Z}}{\partial \delta_{j}}=-\left|V_{i}^{3}\right|\left|V_{j}\right|\left|Y_{i j}\right| \cos \left(\theta_{i j}-\delta_{i}+\delta_{j}\right) j \neq i \\
& \frac{\partial Q_{Z}}{\partial\left|V_{i}\right|}=-4\left|V_{i}^{3}\right|\left|Y_{i i}\right| \sin \theta_{i i}-\sum_{j \neq i} 3\left|V_{i}^{2}\left\|V_{j}\right\| Y_{i j}\right| \sin \left(\theta_{i j}-\delta_{i}+\delta_{j}\right) \\
& \frac{\partial Q_{Z}}{\partial\left|V_{j}\right|}=-\left|V_{i}^{3}\right|\left|Y_{i j}\right| \sin \left(\theta_{i j}-\delta_{i}+\delta_{j}\right) j \neq i
\end{aligned}
$$

\subsection{Constant Current (I) Incorporated with Newton-Raphson Method:}

Newton Raphson [11] represents both active and reactive powers and incorporated in equation (7) and (8).

$$
\begin{aligned}
& P_{I}=\left[\sum_{j=i}^{n}\left|V_{i}\right|\left|V_{j}\right|\left|Y_{i j}\right| \cos \left(\theta_{i j}-\delta_{i}+\delta_{j}\right)\right] V_{i} \\
& Q_{I}=\left[-\sum_{j=i}^{n}\left|V_{i}\right|\left|V_{j}\right|\left|Y_{i j}\right| \sin \left(\theta_{i j}-\delta_{i}+\delta_{j}\right)\right] V_{i}
\end{aligned}
$$

The diagonal and the off-diagonal elements of active power are represented below.

$$
\begin{aligned}
& \frac{\partial P_{I}}{\partial \delta_{i}}=\sum_{j \neq i}\left|V_{i}^{2}\right|\left|V_{j}\right|\left|Y_{i j}\right| \sin \left(\theta_{i j}-\delta_{i}+\delta_{j}\right) \\
& \frac{\partial P_{I}}{\partial \delta_{j}}=-\left|V_{i}^{2}\right|\left|V_{j}\right|\left|Y_{i j}\right| \sin \left(\theta_{i j}-\delta_{i}+\delta_{j}\right) j \neq i
\end{aligned}
$$




$$
\begin{aligned}
& \frac{\partial P_{I}}{\partial\left|V_{i}\right|}=3\left|V_{i}^{2}\right|\left|Y_{i i}\right| \cos \theta_{i i}+\sum_{j \neq i} 2\left|V_{i}\right|\left|V_{j}\right|\left|Y_{i j}\right| \cos \left(\theta_{i j}-\delta_{i}+\delta_{j}\right) \\
& \frac{\partial P_{I}}{\partial\left|V_{j}\right|}=\left|V_{i}^{2}\right|\left|Y_{i j}\right| \cos \left(\theta_{i j}-\delta_{i}+\delta_{j}\right) j \neq i
\end{aligned}
$$

The diagonal and the off-diagonal elements of reactive power are represents below.

$$
\begin{aligned}
& \frac{\partial Q_{I}}{\partial \delta_{i}}=\sum_{j \neq i}\left|V_{i}^{2}\right|\left|V_{j}\right|\left|Y_{i j}\right| \cos \left(\theta_{i j}-\delta_{i}+\delta_{j}\right) \\
& \frac{\partial Q_{I}}{\partial \delta_{j}}=-\left|V_{i}^{2}\right|\left|V_{j}\right|\left|Y_{i j}\right| \cos \left(\theta_{i j}-\delta_{i}+\delta_{j}\right) j \neq i \\
& \frac{\partial Q_{I}}{\partial\left|V_{i}\right|}=3\left|V_{i}^{2}\right|\left|Y_{i i}\right| \sin \theta_{i i}-\sum_{j \neq i} 2\left|V_{i}\right|\left|V_{j}\right|\left|Y_{i j}\right| \sin \left(\theta_{i j}-\delta_{i}+\delta_{j}\right) \\
& \frac{\partial Q_{I}}{\partial\left|V_{j}\right|}=-\left|V_{i}^{2} \| Y_{i j}\right| \sin \left(\theta_{i j}-\delta_{i}+\delta_{j}\right) j \neq i
\end{aligned}
$$

\subsection{Constant Power (P) Incorporated with Newton-Raphson Method:}

Newton Raphson [11] represents both active and reactive powers and incorporated in equation (9) and (10).

$$
\begin{aligned}
& P_{P}=\left[\sum_{j=i}^{n}\left|V_{i}\right|\left|V_{j}\right|\left|Y_{i j}\right| \cos \left(\theta_{i j}-\delta_{i}+\delta_{j}\right)\right] \\
& Q_{P}=\left[-\sum_{j=i}^{n}\left|V_{i}\right|\left|V_{j}\right|\left|Y_{i j}\right| \sin \left(\theta_{i j}-\delta_{i}+\delta_{j}\right)\right]
\end{aligned}
$$

The diagonal and the off-diagonal elements of active power are represented below.

$$
\begin{aligned}
& \frac{\partial P_{P}}{\partial \delta_{i}}=\sum_{j \neq i}\left|V_{i}\right|\left|V_{j}\right|\left|Y_{i j}\right| \sin \left(\theta_{i j}-\delta_{i}+\delta_{j}\right) \\
& \frac{\partial P_{P}}{\partial \delta_{j}}=-\left|V_{i}\right|\left|V_{j}\right|\left|Y_{i j}\right| \sin \left(\theta_{i j}-\delta_{i}+\delta_{j}\right) j \neq i \\
& \frac{\partial P_{P}}{\partial\left|V_{i}\right|}=2\left|V_{i}\right|\left|Y_{i i}\right| \cos \theta_{i i}+\sum_{j \neq i}\left|V_{j}\right|\left|Y_{i j}\right| \cos \left(\theta_{i j}-\delta_{i}+\delta_{j}\right) \\
& \frac{\partial P_{P}}{\partial\left|V_{j}\right|}=\left|V_{i}\right|\left|Y_{i j}\right| \cos \left(\theta_{i j}-\delta_{i}+\delta_{j}\right) j \neq i
\end{aligned}
$$

The diagonal and the off-diagonal elements of reactive power are represented below.

$$
\begin{aligned}
& \frac{\partial Q_{P}}{\partial \delta_{i}}=\sum_{j \neq i}\left|V_{i}\right|\left|V_{j}\right|\left|Y_{i j}\right| \cos \left(\theta_{i j}-\delta_{i}+\delta_{j}\right) \\
& \frac{\partial Q_{P}}{\partial \delta_{j}}=\left|V_{i}\right|\left|V_{j}\right|\left|Y_{i j}\right| \cos \left(\theta_{i j}-\delta_{i}+\delta_{j}\right) j \neq i \\
& \frac{\partial Q_{P}}{\partial\left|V_{i}\right|}=-2\left|V_{i}\right|\left|Y_{i i}\right| \sin \theta_{i i}-\sum_{j \neq i}\left|V_{j}\right|\left|Y_{i j}\right| \sin \left(\theta_{i j}-\delta_{i}+\delta_{j}\right)
\end{aligned}
$$




$$
\frac{\partial Q_{P}}{\partial\left|V_{j}\right|}=-\left|V_{i}\right|\left|Y_{i j}\right| \sin \left(\theta_{i j}-\delta_{i}+\delta_{j}\right) j \neq i
$$

\subsection{ZIP Incorporated With Newton-Raphson Method:}

Newton Raphson [11] represents both active and reactive powers and incorporated in equation (3) and (4).

In this ZIP load model, the main parameters are $P_{1}=P_{2}=P_{3}=0.333$, $Q_{1}=Q_{2}=Q_{3}=0.333$. Substitute these parameters in below equations.

$P_{\text {ZIP }}=\left[\sum_{j=1}^{n}\left|V_{i}\right|\left|V_{j}\right|\left|Y_{i j}\right| \cos \left(\theta_{i j}-\delta_{i}+\delta_{j}\right)\right]\left[p_{1} \bar{v}^{2}+p_{2} \bar{v}+p_{3}\right]$
$=P_{1}\left[\sum_{j=1}^{n}\left|V_{i}^{3}\left\|V_{j}\right\| Y_{i j}\right| \cos \left(\theta_{i j}-\delta_{i}+\delta_{j}\right)\right]+P_{2}\left[\sum_{j=1}^{n}\left|V_{i}^{2} \| V_{j}\right|\left|Y_{i j}\right| \cos \left(\theta_{i j}-\delta_{i}+\delta_{j}\right)\right]+$

$P_{3}\left[\sum_{j=1}^{n}\left|V_{i}\right|\left|V_{j}\right|\left|Y_{i j}\right| \cos \left(\theta_{i j}-\delta_{i}+\delta_{j}\right)\right]$

$Q_{Z I P}=\left[-\sum_{j=1}^{n}\left|V_{i}\right|\left|V_{j}\right|\left|Y_{i j}\right| \sin \left(\theta_{i j}-\delta_{i}+\delta_{j}\right)\right]\left[q_{1} \bar{v}+q_{2} \bar{v}+q_{3}\right]$

$=P_{1}\left[-\sum_{j=1}^{n}\left|V_{i}^{3} \| V_{j}\right|\left|Y_{i j}\right| \sin \left(\theta_{i j}-\delta_{i}+\delta_{j}\right)\right]+P_{2}\left[-\sum_{j=1}^{n}\left|V_{i}^{2}\left\|V_{j}\right\| Y_{i j}\right| \sin \left(\theta_{i j}-\delta_{i}+\delta_{j}\right)\right]+$

$P_{3}\left[-\sum_{j=1}^{n}\left|V_{i}\right|\left|V_{j} \| Y_{i j}\right| \sin \left(\theta_{i j}-\delta_{i}+\delta_{j}\right)\right]$

The diagonal and the off-diagonal elements of active power are represented below.

$\frac{\partial P_{Z I P}}{\partial \delta_{i}}=\left\{P_{1}\left[\sum_{j \neq i}\left|V_{i}^{3} \| V_{j}\right|\left|Y_{i j}\right| \sin \left(\theta_{i j}-\delta_{i}+\delta_{j}\right)\right]+P_{2}\left[\sum_{j \neq i}\left|V_{i}^{2}\left\|V_{j}\right\| Y_{i j}\right| \sin \left(\theta_{i j}-\delta_{i}+\delta_{j}\right)\right]+\right.$

$\left.P_{3}\left[\sum_{j \neq i}\left|V_{i}\right|\left|V_{j}\right|\left|Y_{i j}\right| \sin \left(\theta_{i j}-\delta_{i}+\delta_{j}\right)\right]\right\}$

$\frac{\partial P_{Z I P}}{\partial \delta_{j}}=\left\{P_{1}\left[-\left|V_{i}^{3}\right|\left|V_{j}\right|\left|Y_{i j}\right| \sin \left(\theta_{i j}-\delta_{i}+\delta_{j}\right)\right]+P_{2}\left[-\left|V_{i}^{2} \| V_{j}\right|\left|Y_{i j}\right| \sin \left(\theta_{i j}-\delta_{i}+\delta_{j}\right)\right]+\right.$

$\left.P_{3}\left[-\left|V_{i}\right|\left|V_{j}\right|\left|Y_{i j}\right| \sin \left(\theta_{i j}-\delta_{i}+\delta_{j}\right)\right]\right\} j \neq i$ 


$$
\begin{aligned}
& \frac{\partial P_{Z I P}}{\partial\left|V_{i}\right|}=\left\{P_{1}\left[4\left|V_{i}^{3}\right|\left|Y_{i i}\right| \cos \theta_{i i}+\sum_{j \neq i} 3\left|V_{i}^{2} \| V_{j}\right|\left|Y_{i j}\right| \cos \left(\theta_{i j}-\delta_{i}+\delta_{j}\right)\right]+\right. \\
& P_{2}\left[3\left|V_{i}^{2}\right|\left|Y_{i i}\right| \cos \theta_{i i}+\sum_{j \neq i} 2\left|V_{i}^{2}\right|\left|V_{j}\right|\left|Y_{i j}\right| \cos \left(\theta_{i j}-\delta_{i}+\delta_{j}\right)\right]+ \\
& \left.P_{3}\left[2\left|V_{i} \| Y_{i i}\right| \cos \theta_{i i}+\sum_{j \neq i}\left|V_{j}\right|\left|Y_{i j}\right| \cos \left(\theta_{i j}-\delta_{i}+\delta_{j}\right)\right]\right\} \\
& \frac{\partial P_{Z I P}}{\partial\left|V_{j}\right|}=\left\{P_{1}\left[\left|V_{i}^{3} \| Y_{i j}\right| \cos \left(\theta_{i j}-\delta_{i}+\delta_{j}\right)\right]+P_{2}\left[\left|V_{i}^{2} \| Y_{i j}\right| \cos \left(\theta_{i j}-\delta_{i}+\delta_{j}\right)\right]+\right. \\
& \left.P_{3}\left[\left|V_{i}\right|\left|Y_{i j}\right| \cos \left(\theta_{i j}-\delta_{i}+\delta_{j}\right)\right]\right\} j \neq i
\end{aligned}
$$

The diagonal and the off-diagonal elements of reactive power are represented below.

$$
\begin{aligned}
& \frac{\partial Q_{\text {ZIP }}}{\partial \delta_{i}}=\left\{P_{1}\left[\sum_{j \neq i}\left|V_{i}^{3} \| V_{j}\right|\left|Y_{i j}\right| \cos \left(\theta_{i j}-\delta_{i}+\delta_{j}\right)\right]+P_{2}\left[\sum_{j \neq i}\left|V_{i}^{2}\right|\left|V_{j}\right|\left|Y_{i j}\right| \cos \left(\theta_{i j}-\delta_{i}+\delta_{j}\right)\right]\right. \\
& \left.P_{3}\left[\sum_{j \neq i}\left|V_{i}\right|\left|V_{j}\right|\left|Y_{i j}\right| \cos \left(\theta_{i j}-\delta_{i}+\delta_{j}\right)\right]\right\} \\
& \frac{\partial Q_{\text {ZIP }}}{\partial \delta_{j}}=\left\{P_{1}\left[-\left|V_{i}^{3} \| V_{j}\right|\left|Y_{i j}\right| \cos \left(\theta_{i j}-\delta_{i}+\delta_{j}\right)\right]+P_{2}\left[-\left|V_{i}^{2}\right|\left|V_{j}\right|\left|Y_{i j}\right| \cos \left(\theta_{i j}-\delta_{i}+\delta_{j}\right)\right]+\right. \\
& \left.P_{3}\left[-\left|V_{i}\right|\left|V_{j}\right|\left|Y_{i j}\right| \cos \left(\theta_{i j}-\delta_{i}+\delta_{j}\right)\right]\right\} \\
& \frac{\partial Q_{\text {ZIP }}}{\partial\left|V_{i}\right|}=\left\{P_{1}\left[-4\left|V_{i}^{3}\right|\left|Y_{i i}\right| \sin \theta_{i i}-\sum_{j \neq i} 3\left|V_{i}^{2} \| V_{j}\right|\left|Y_{i j}\right| \sin \left(\theta_{i j}-\delta_{i}+\delta_{j}\right)\right]+\right. \\
& P_{2}\left[-3\left|V_{i}^{2}\right|\left|Y_{i i}\right| \sin \theta_{i i}-\sum_{j \neq i} 2\left|V_{i}^{2}\left\|V_{j}\right\| Y_{i j}\right| \sin \left(\theta_{i j}-\delta_{i}+\delta_{j}\right)\right]+ \\
& \left.P_{3}\left[-2\left|V_{i} \| Y_{i i}\right| \sin \theta_{i i}-\sum_{j \neq i}\left|V_{j}\right|\left|Y_{i j}\right| \sin \left(\theta_{i j}-\delta_{i}+\delta_{j}\right)\right]\right\} \\
& \frac{\partial Q_{\text {ZIP }}}{\partial\left|V_{j}\right|}=\left\{P_{1}\left[-\left|V_{i}^{3} \| Y_{i j}\right| \sin \left(\theta_{i j}-\delta_{i}+\delta_{j}\right)\right]+P_{2}\left[-\left|V_{i}^{2} \| Y_{i j}\right| \sin \left(\theta_{i j}-\delta_{i}+\delta_{j}\right)\right]+\right. \\
& \left.P_{3}\left[-\left|V_{i}\right|\left|Y_{i j}\right| \sin \left(\theta_{i j}-\delta_{i}+\delta_{j}\right)\right]\right\} j \neq i
\end{aligned}
$$

From the constant impedance (z), constant current (I), constant power (P), and ZIP incorporated with Newton Raphson method, and we convert equations into the Jacobian matrix. The Jacobian matrix gives the linearized relationship between small changes in voltage angle and voltage magnitude with the small changes in real and reactive power.

$$
\left[\begin{array}{c}
\Delta P \\
\Delta Q
\end{array}\right]=\left[\begin{array}{ll}
J_{1} & J_{2} \\
J_{3} & J_{4}
\end{array}\right]\left[\begin{array}{c}
\Delta \delta \\
\Delta|V|
\end{array}\right]
$$

The terms and are the difference between the scheduled and calculated values, known as the power residual given by 


$$
\begin{aligned}
\Delta P_{i}^{k} & =P_{i}^{s c h}-P_{i}^{k} \\
\Delta Q_{i}^{k} & =Q_{i}^{s c h}-Q_{i}^{k}
\end{aligned}
$$

The new estimates for bus voltages are

$$
\begin{gathered}
\delta_{i}^{(k+1)}=\delta_{i}^{k}+\Delta \delta_{i}^{(k)} \\
\left|V_{i}^{(k+1)}\right|=\left|V_{i}^{(k)}\right|+\Delta\left|V_{i}^{k}\right|
\end{gathered}
$$

\section{Proposed Algorithm}

The computational methodology has been carried out through the following steps.

Step 1: Read line and bus data of the given system and assumes that system angle, load (MW \& MVAR) and generator (MW \& MVAR, Qmin \& Qmax) data are constant.

Step 2: Carry out the load flow studies using constant $\mathrm{Z}$ load model using equation (5) \& (6).

Step 3: Carry out the load flow studies using constant I load model using equation (7) \& (8).

Step 4: Carry out the load flow studies using P load model using equation (9) \& (10).

Step 5: Carry out the load flow studies using ZIP load modeling equation (3) \& (4).

Step 6: Calculate voltage magnitudes, voltage angles, active and reactive power flows, Active and reactive power losses, number of iterations, power mismatch, for step 2 to step 6 .

\section{Case Study}

A standard 2-machine 5-bus system is considered for the simulation purpose. This system consists of a slack bus, 1-generator, and 7-transmission lines. Newton Raphson load flow technique is used for understanding the system performance with all the load models.

The voltage magnitude for P-Alone, Z-Alone, I-Alone, and ZIP are given in Table 5.1. Compared to various load models, the constant impedance $(\mathrm{Z})$ load model is affecting more on the system. The constant power $(\mathrm{P})$ model has a very low effect on the power system.

Table 5.1. Voltage Magnitudes

\begin{tabular}{|c|c|c|c|c|}
\hline Bus No & Z-Alone & I-Alone & P-Alone & ZIP \\
\hline 1 & 1.06 & 1.06 & 1.06 & 1.06 \\
\hline 2 & 1 & 1 & 1 & 1 \\
\hline 3 & 0.9858 & 0.9866 & 0.9872 & 0.9865 \\
\hline 4 & 0.9826 & 0.9834 & 0.9841 & 0.9834 \\
\hline 5 & 0.9694 & 0.9706 & 0.9717 & 0.9706 \\
\hline
\end{tabular}

The voltage angles for P-Alone, Z-Alone, I-Alone, and ZIP are given in Table 5.2. Compared to various load models, the constant impedance $(Z)$ load model is affecting more on the system. The constant power $(\mathrm{P})$ model has a very low effect on the power system. 
Table 5.2. Voltage Angles

\begin{tabular}{|c|c|c|c|c|}
\hline Bus No & Z-Alone & I-Alone & P-Alone & ZIP \\
\hline 1 & 0 & 0 & 0 & 0 \\
\hline 2 & -2.2447 & -2.1474 & -2.0612 & -2.1504 \\
\hline 3 & -4.8885 & -4.755 & -4.6367 & -4.7602 \\
\hline 4 & -5.2332 & -5.0868 & -4.957 & -5.0924 \\
\hline 5 & -6.1591 & -5.9503 & -5.7649 & -5.9563 \\
\hline
\end{tabular}

The Active Power Flows for P-Alone, Z-Alone, I-Alone, and ZIP are given in Table 5.3. Compared to various load models, the constant impedance $(\mathrm{Z})$ load model is affecting more on the system. The constant power $(\mathrm{P})$ model has a very low effect on the power system.

Table 5.3. Active Power Flows

\begin{tabular}{|c|c|c|c|c|}
\hline Line No & Z-Alone & I-Alone & P-Alone & ZIP \\
\hline 1 & 0.9447 & 0.9175 & 0.8933 & 0.9183 \\
\hline 2 & 0.4369 & 0.4269 & 0.4179 & 0.4273 \\
\hline 3 & 0.2527 & 0.2485 & 0.2447 & 0.2487 \\
\hline 4 & 0.2873 & 0.2819 & 0.2771 & 0.2822 \\
\hline 5 & 0.5785 & 0.5616 & 0.5466 & 0.5621 \\
\hline 6 & 0.2065 & 0.1998 & 0.1939 & 0.2 \\
\hline 7 & 0.0742 & 0.0698 & 0.066 & 0.0699 \\
\hline
\end{tabular}

The Reactive Power Flows for P-Alone, Z-Alone, I-Alone, and ZIP are given in Table 5.4. Compared to various load models, the constant power $(\mathrm{P})$ load model is affecting more on the system. The constant impedance $(\mathrm{P})$ model has a very low effect on the power system.

Table 5.4. Reactive Power Flows

\begin{tabular}{|c|c|c|c|c|}
\hline Line No & Z-Alone & I-Alone & P-Alone & ZIP \\
\hline 1 & 0.7249 & 0.7328 & 0.740 & 0.7326 \\
\hline 2 & 0.1697 & 0.1689 & 0.1682 & 0.169 \\
\hline 3 & -0.0197 & -0.0226 & -0.0252 & -0.0224 \\
\hline 4 & -0.0118 & -0.0147 & -0.0172 & -0.0146 \\
\hline 5 & 0.0663 & 0.0606 & 0.0556 & 0.0608 \\
\hline 6 & 0.027 & 0.0279 & 0.0286 & 0.0278 \\
\hline 7 & 0.006 & 0.0055 & 0.0052 & 0.0055 \\
\hline
\end{tabular}

The Total power mismatch for P-Alone, Z-Alone, I-Alone, and ZIP are given in Fig 5.1. Compared to various load models, the constant impedance $(\mathrm{P})$ load model is affecting more on the system. The constant power $(\mathrm{P})$ model has a very low effect on the power system. 


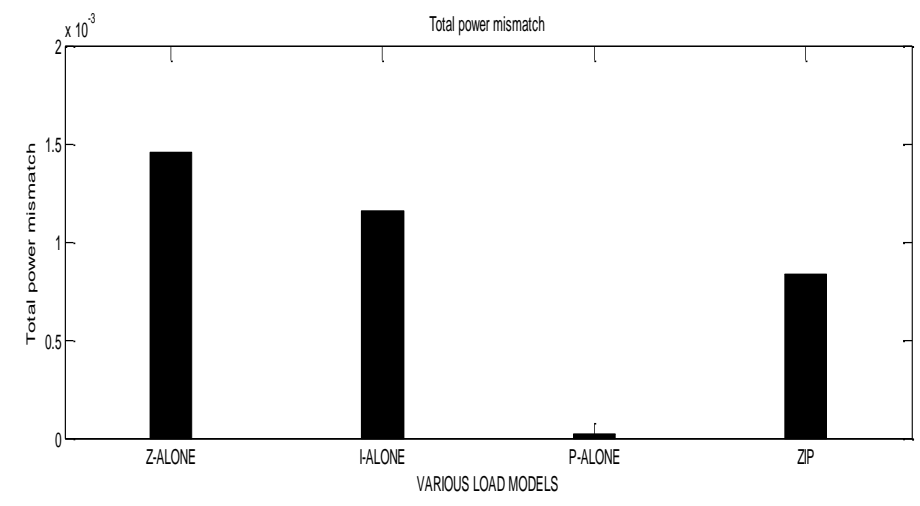

Figure 5.1. Total Power Mismatch

The Active Power Loss for P-Alone, Z-Alone, I-Alone, and ZIP are given in Table 5.5. Compared to various load models, the constant impedance $(\mathrm{P})$ load model is affecting more on the system. The constant power $(\mathrm{P})$ model has a very low effect on the power system.

Table 5.5. Active Power Loss

\begin{tabular}{|c|c|c|c|c|}
\hline Line No & Z-Alone & I-Alone & P-Alone & ZIP \\
\hline 1 & 0.0261 & 0.0254 & 0.0249 & 0.0255 \\
\hline 2 & 0.0164 & 0.0157 & 0.0152 & 0.0158 \\
\hline 3 & 0.0038 & 0.0037 & 0.0036 & 0.0037 \\
\hline 4 & 0.005 & 0.0048 & 0.0046 & 0.0048 \\
\hline 5 & 0.0137 & 0.0128 & 0.0122 & 0.0129 \\
\hline 6 & 0.0005 & 0.0004 & 0.0004 & 0.0004 \\
\hline 7 & 0.0005 & 0.0005 & 0.0004 & 0.0005 \\
\hline
\end{tabular}

The Reactive Power Loss for P-Alone, Z-Alone, I-Alone, and ZIP are given in Table 5.6. Compared to various load models, the constant impedance $(\mathrm{P})$ load model is affecting more on the system. The constant power $(\mathrm{P})$ model has a very low effect on the power system.

Table 5.6. Reactive Power Loss

\begin{tabular}{|c|c|c|c|c|}
\hline Line No & Z-Alone & I-Alone & P-Alone & ZIP \\
\hline 1 & 0.0147 & 0.0126 & 0.0109 & 0.0127 \\
\hline 2 & -0.0032 & -0.0052 & -0.0069 & -0.0051 \\
\hline 3 & -0.0279 & -0.0284 & -0.0287 & -0.0283 \\
\hline 4 & -0.0244 & -0.025 & -0.0255 & -0.025 \\
\hline 5 & 0.0119 & 0.0094 & 0.0073 & 0.0095 \\
\hline 6 & -0.018 & -0.0181 & -0.0182 & -0.0181 \\
\hline 7 & -0.046 & -0.0463 & -0.0465 & -0.0463 \\
\hline
\end{tabular}

The Reactive Power Generation for P-Alone, Z-Alone, I-Alone, and ZIP are given in Table 5.7. Compared to various load models, the constant power $(\mathrm{P})$ load model is affecting more on the system. The constant impedance $(\mathrm{P})$ model has a very low effect on the power system. 
Table 5.7. Reactive Power Generation

\begin{tabular}{|c|c|c|c|c|}
\hline Bus No & Z-Alone & I-Alone & P-Alone & ZIP \\
\hline 1 & 0.8947 & 0.9017 & 0.9082 & 0.9016 \\
\hline 2 & -0.6752 & -0.697 & -0.7159 & -0.6962 \\
\hline
\end{tabular}

The Total Active Power Loss for P-Alone, Z-Alone, I-Alone, and ZIP are given in Fig 5.2. Compared to various load models, the constant impedance $(\mathrm{Z})$ load model is affecting more on the system. The constant power $(\mathrm{P})$ model has a very low effect on the power system.

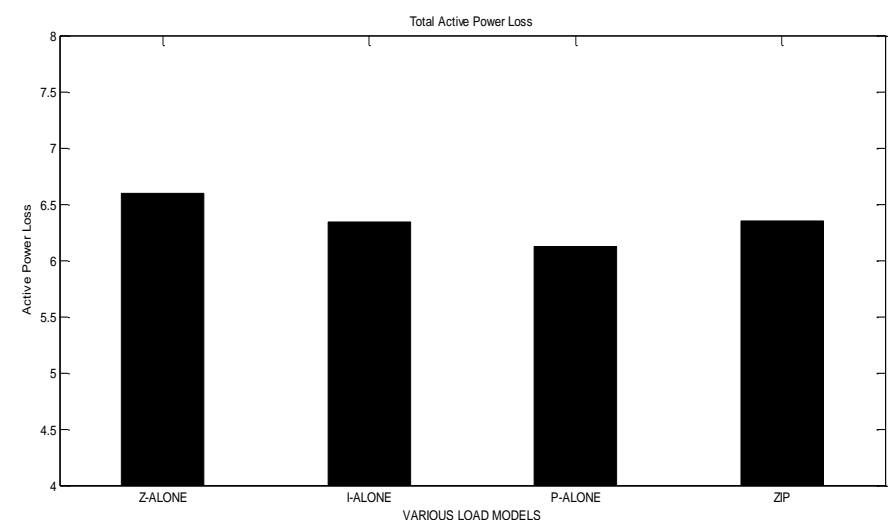

Figure 5.2. Total Active Power Loss

The Total Reactive Power Loss for P-Alone, Z-Alone, I-Alone, and ZIP are given in Figure 5.3. Compared to various load models, the constant power $(\mathrm{P})$ load model is affecting more on the system. The constant impedance $(\mathrm{Z})$ model has a very low effect on the power system.

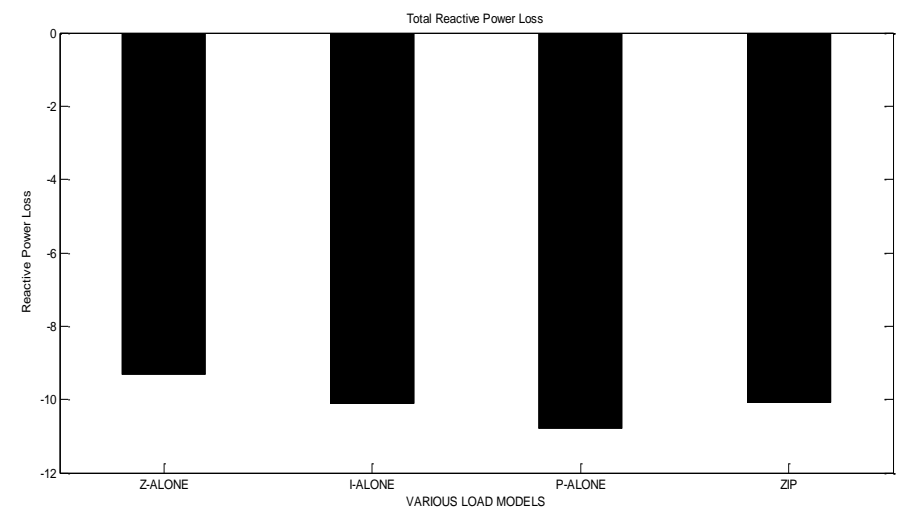

Figure 5.3. Total Reactive Power Loss

The Active Power Generation for P-Alone, Z-Alone, I-Alone, and ZIP are given in Figure 5.4. Compared to various load models, the constant impedance $(\mathrm{Z})$ load model is affecting more on the system. The constant power $(\mathrm{P})$ model has a very low effect on the power system. 


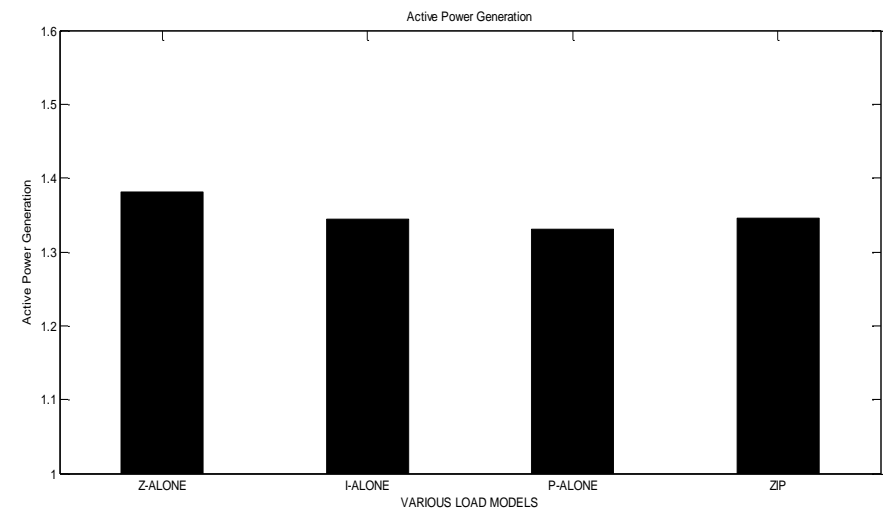

Figure 5.4. Active Power Generation

\section{Conclusion}

An investigation of the effects of incorporating ZIP load modeling is shown in this paper. The active and reactive power in the transmission lines is given. The voltage at each bus is given. The results for Impedance (Z), Current (I), Power (P), ZIP is incorporated in Newton Raphson method. The comparison of all the methods is shown. $Z$ alone system has high losses compared to any other method and $\mathrm{P}$ alone system is used as the best load model as it has fewer losses in the system.

\section{Acknowledgments}

The authors are thankful to Dr. T. Nageswara Prasad, HOD and advanced renewable research lab, Department of EEE Sree Vidyanikethan Engineering College, Tirupati for the constant encouragement and providing the facilities to complete this work.

\section{References}

[1] C.-H. Gu, Q. Ai and J. Wu, "A Study of Effect of Different Static Load Models and System Operating Constraints on Static Voltage Stability", Proceeding of 5th WSEAS/IASME international Conference on system theory and scientific computation, malta, (2005) September, pp. 44-49.

[2] A. Bokhari, A. Alkan, R. Dogan, M. Diaz-Aguiló, F. de León, D. Czarkowski and Z. Zabar, "Experimental Determination of the ZIP Coefficients for Modern Residential, Commercial, and Industrial Loads", IEEE Transactions on Power Delivery, (2014) June, vol. 29.

[3] B.-K. Choi, H. D. Chiang, Y. Li, Y. T. Chen, D. H. Huang and M. G. Lauby, "Development of composite load models of power systems using on-line measurement data", Journal of Electrical Engineering and Technology, vol. 1, no. 2, (2006), pp. 161-169.

[4] Shengyang, "Modeling Power System Load Using Intelligent Methods", Ph. D Thesis, (2011).

[5] P. Kundur, "Power System Stability and Control", New York: Mc- Graw-Hill, (1994), pp. 17-279.

[6] D.-Hyun Chung, "A Study on the Measurement Based Approach Techniques for Static Load Modeling", International Journal of Computer and Information Technology (IJCIT), vol. 03, iss. 02, (2014) March.

[7] A. Borghetti, R. Caldon, A. Mari and C. A. Nucci, "On Dynamic Load Models for Voltage Stability Studies", IEEE Transactions on Power Syst., vol. 12, (1997) November.

[8] H. H. Chang, J. Wen, Y. P. Tsai and W. J. Lee, "A New Measurement Method for Power Signatures of Non-intrusive Demand Monitoring and Load Identification", IEEE Transactions on Industry Applications, vol. 48, no. 2, (2012), pp. 764-771.

[9] S. Kamel and M. K. El-nemr, "Hybrid Power And Current Mismatches Newton-raphson Load-flow Analysis For Solving Power Systems With Voltage Controlled Devices", International Middle East Power System Conference (MEPCON'10), (2010) December, pp. 1-6.

[10] M. Sadeghi and G. A. Sarvi, "Determination of ZIP parameters with least squares optimization method", in Elect. Power Energy Conf., (2009), pp. 1-6.

[11] H. Saadat, "Power System Analysis", Third Reprint Tata Mcgraw-hill, (2003). 


\section{Authors}

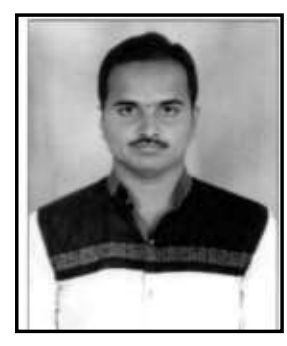

Seshapalli Sairam was born in West Godavari (A.P), India in 1995. He received his B.Tech in Electrical and Electronics Engineering from Ramachandra College of Engineering in 2016. He is a PG Scholar in the Department of Electrical and Electronics Engineering at Sree Vidyanikethan Engineering College, Tirupathi (India).

His area of research includes load modeling, voltage stability studies, security analysis.

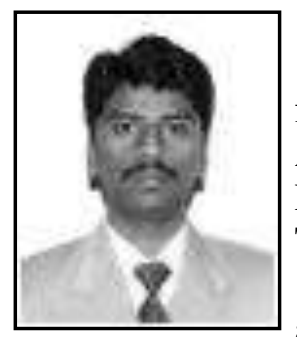

Suresh Babu Daram was born in Vijayawada (A.P), India in 1985. He received his B.Tech in Electrical \& Electronics engineering from JNTU in 2006 and M.Tech degree in Power Systems Engg from ANU, in 2009. He is a Research Scholar in the Department of Electrical \& Electronics Engineering at the Visvesvaraya Technological University Belgaum (India).

He was Assistant Professor in the Dept. of Electrical \& Electronics at GGITM Bhopal from 2009-2015. Currently he is Asst. Prof in Dept. of Electrical \& Electronics, Sree Vidyanikethan Engineering College, Tirupati (A.P), India. He has published 32 national/ International journal / conference papers. His research interests include energy management systems, power system optimization, and voltage instability studies incorporating FACTS controllers and security analysis.

Mr. Suresh is a member of IEEE, AMIE (India), IAENG, CSTA, IACSIT, IRED and Student Member-ASTM.

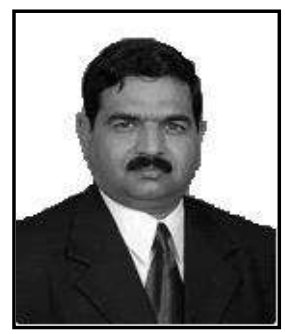

P. S. Venkataramu was born in India on June 20, 1961. He received his Graduation in Electrical Engineering from the Institute of Engineers (India), M. Tech degree in Power Systems from Mysore University (India) and Ph.D. in 2007 from Visvesvaraya Technological University, Belgaum (India).

He was employed as an Electrical Engineer in the Goa state Electricity Department and worked for 15 years in various capacities. He was primarily involved in carrying power system operational and planning studies for the regional grid system. He was also a visiting faculty in the Goa college of Engineering. From 1997 to 2007, he worked as a faculty in various positions at School of Electrical Sciences, Vellore Institute of Technology, Vellore, India. From 2007 to 2015 he was a Professor in Electrical Engineering Department and Principal of Gyan Ganga Institute of Technology and Management, Bhopal (India). Currently he is Director -Internal Quality at REVA University, Bangalore. He has several national and international publications. His research interest includes AI application to power system and distribution system automation.

Dr. P. S. Venkataramu is a Fellow of the institution of engineers (India), Indian society for technical education, and System society of India. 


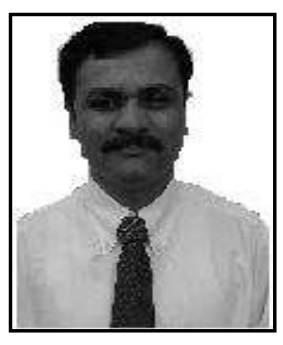

M. S. Nagaraj completed his B.E degree in Electrical \& Electronics in the year 1986 from Government BDT College of Engineering, Davangere. Master Engineering, Mysore in the year $1991 \& 2007$ respectively.

He has teaching experience of 25 years in Under Graduate College. At present, he is working as Professor \& Head in the Department of Electrical \& Electronics Engg, BIET, Davangere. He has published more than 30 papers in national/ International journal /conference papers. His research interest includes AI application to the power system.

Dr. M. S. Nagaraj is a member of Indian Society for Technical Education and Institution of Engineers (India). 